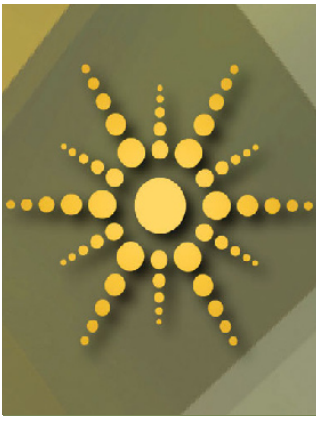

\section{BREWing better broader impacts}

\author{
nsf.gov/od/oia/special/broaderimpacts
}

\section{By Anne Lynn Gillian-Daniel, Matthew D. Stilwell, Nicholas L. Abbott, and Wendy C. Crone}

$T^{n}$ he US National Science Foundation (NSF) requires all grant-funded research projects to include a "Broader Impacts" component, that is, the potential to benefit society and contribute to the achievement of specific, desired societal outcomes. ${ }^{1,2}$ However, achieving the broader impacts mission with full participation from all members is a significant challenge faced by research centers, as well as other large groups of researchers who work together in departments, laboratory groups, and training grants (groups of trainees at an institution).

Large, NSF-funded research centers, such as the Materials Research Science and Engineering Centers (MRSECs), have a unique opportunity to achieve broader impacts by using researchers' collective, interdisciplinary expertise to assist in both the development of novel products and programs and the evaluation of their scientific content. This type of engagement is particularly valuable to the creation of innovative and accurate educational products that can engage people outside of the research field with concepts related to cutting-edge research, help them to understand the importance of science and engineering, and stimulate the future workforce's interest in science, technology, engineering, and mathematics (STEM).

To engage participation of all center members in educational products development, the MRSEC at the University of Wisconsin-Madison created the Breakthrough Research and Education Workshop (BREW). BREW combines cutting-edge research presentations with an education workshop focused on the development of novel, research-inspired educational content.

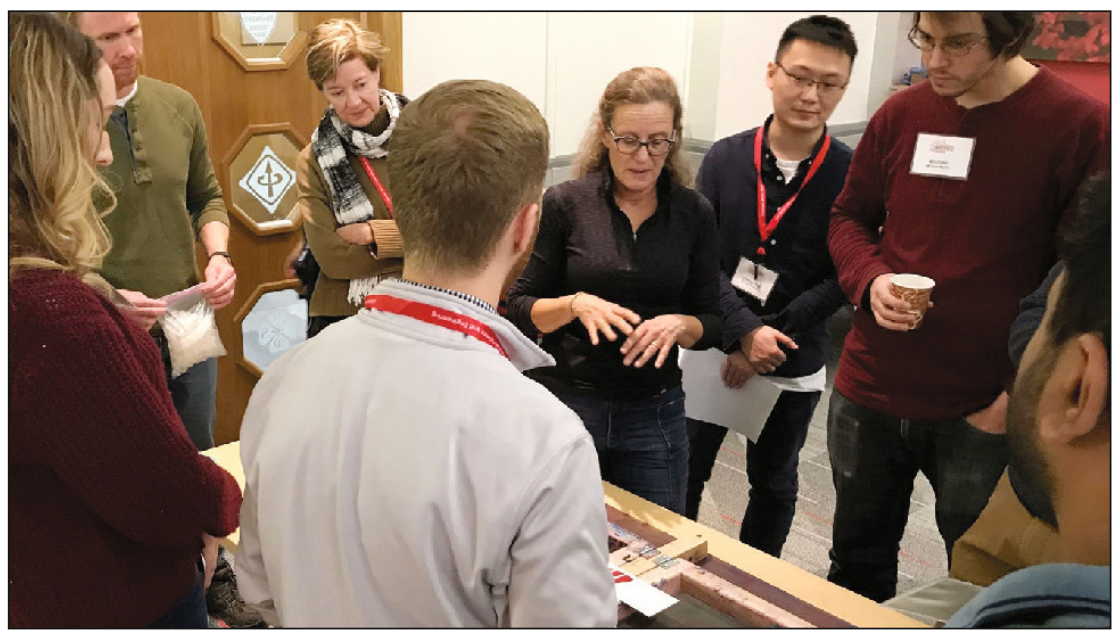

University of Wisconsin-Madison MRSEC members engage with and evaluate an educational activity that is under development during the 2019 BREW.

\section{The structure of BREW}

Since 2012, the Wisconsin MRSEC has held an annual BREW to enhance collaboration and build community among all center members, including faculty, staff, postdoctoral research associates, and graduate research assistants. BREW is a half-day, all-member event, beginning with 3-5 research highlight presentations followed by an interactive educational workshop. Faculty members introduce the research, and then their graduate students and postdoctoral associates present short vignettes on their contributions to that area of research. A primary function of the MRSEC program is to train early-career researchers, and BREW provides an excellent opportunity for these researchers to practice their scientific presentation skills. The goal of the interactive education workshop is to leverage the research talks as inspiration for new educational activities or expansion of existing activities. The workshop is facilitated by the Center's education/outreach $(\mathrm{E} / \mathrm{O})$ faculty and staff, and is typically scheduled for the same length of time as all of the research presentations together, emphasizing the importance of education and outreach in the Center.

\section{BREW E/O workshop outcomes}

BREW's E/O workshop is designed as a brainstorming session, which typically yields a large number of ideas. Additionally, the workshop serves the larger purpose of emphasizing the importance of education and outreach in the Center's mission and getting faculty, students, and staff thinking about how educational activities are developed. This workshop typically leads to further discussions throughout the year about how the Center's research can be translated into public outreach activities. Ultimately, a subset of the ideas from the BREW is further developed into public outreach activities or programs.

One example of content that arose from the $2013 \mathrm{BREW}$ E/O workshop is 
a three-dimensional, touch screen simulation called Atom Touch, ${ }^{3}$ which was developed in collaboration with MRSEC faculty member Dane Morgan, University of Wisconsin-Madison, who had the initial idea. Atom Touch was designed to engage middle-school students in learning about how atoms interact with one another and behave under changing physical conditions. The game has been played more than 130,000 times since it was released in February 2018 on the online educational website, BrainPOP.

Other content that arose from the BREW includes a liquid-crystal sensor that undergoes a dramatic visual change from bright to black in the presence of volatile organic compounds, and a board game about how crystals form. The board game was later developed into a digital game called Crystal Cave, ${ }^{4}$ which has been played more than 135,000 times since its release. All of these activities were developed, tested, and refined by the MRSEC's E/O staff in collaboration with researchers.

\section{Effective practices for the BREW E/O workshop}

Based on our experience with the E/O workshops, we have developed a set of effective practices designed to engage researchers in education efforts. These include expecting all members to participate, connecting the $\mathrm{E} / \mathrm{O}$ activities to the broader research mission of the organization, providing activities that require participants to work in diverse teams, engaging in small group work that requires refinement of the ideas generated, presenting top ideas to all participants for further refinement/prioritization, and evaluating the participant experience to enable continuous improvement. See inset box for a detailed list of effective practices.

\section{Impact of the BREW E/O workshop on attendees}

To evaluate the efficacy of the BREW E/O workshop, we distributed surveys following past workshops that led to improvements in subsequent workshop iterations. Data from the 2013 and 2014 workshops are shown in Figure 1. Suggestions the respondents gave for improving the $\mathrm{E} / \mathrm{O}$

\section{Effective practices for the BREW E/O workshop}

1. Begin with a short (5-10-minute) overview of the E/O activities of the Center to orient and update everyone to the work being done in this area.

2. Divide the Center members into diverse teams across research projects and career stages. Diversifying teams facilitates interactions and community building between people working on different research projects, provides opportunities for students and postdocs to network with faculty members, and enables these teams to brainstorm more creatively.

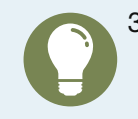

3. Assign a specific brainstorming task to the teams. For example, in previous years, the E/O staff asked the teams to brainstorm a 5-10-minute tabletop activity for middle-school students. Because all Center members participate in public outreach events, attendees are familiar with these types of activities. However, E/O staff must give clear and specific directions for the task, including an overview of what the target audiences are expected to know about the science topic. According to the Next Generation Science Standards, ${ }^{5}$ middleschool students are able to describe the atomic composition of simple molecules; however, these students have not yet been introduced to the periodic table and do not know that properties of elements are connected to the electrons in the outer shell. The overview helps the teams develop content that is accessible and engaging for the target audience.

4. Following the brainstorming session, ask the teams to select their best 2-3 ideas and expand upon them. Provide poster paper, markers, scissors, construction paper, and other supplies for the teams to write, draw, or otherwise represent their ideas. Pose questions such as: What will participants do at the activity? What is interesting or surprising about the activity? What are the materials required to build or make the activity? What concepts are the participants expected to learn? What background will participants need to understand the concept?

5. At the end of the workshop, have each team present their top two ideas to the entire group. Because the BREW is also designed as a fun, communitybuilding event, there can be a friendly competition to select the top idea. Everyone votes on their favorite idea, and the winning team is announced before the event ends.

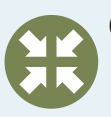

6. Following the workshop, the E/O members collect all of the sketches and written materials that have been created. Feasible ideas are then developed into prototype activities with input from the members of the team who initially proposed it, as well as Center members who are experts in the area covered by the activity. Typically, the workshops produce initial ideas that undergo significant revision during prototype development and field testing.

workshop included giving the workshop more time, building upon existing outreach activities, and having people complete pre-workshop activities to prepare for the BREW E/O workshop. Some suggestions from the evaluation were incorporated into subsequent $\mathrm{E} / \mathrm{O}$ workshops as part of their iterative improvement.

\section{Extensions of the BREW E/O workshop format}

The goals of the BREW E/O workshop can be altered to suit various needs. For example, instead of focusing on new activity development, teams could evaluate activities that are under development. In this case, the workshop format can be similar to the one described earlier, but the session starts with an introduction of the activity and an opportunity for handson engagement. The brainstorming session then focuses on ways to improve and expand the scope of the activity. During the 2017 BREW, researchers play-tested the prototype of a digital game about scientific modeling. The E/O staff and game developers evaluated the attendees' experiences through observation and written surveys. Then the Center members broke into teams to brainstorm ways to improve 
the modeling game and introduce more materials science-specific concepts into the game play. This valuable researcher feedback was incorporated into the game, along with teacher feedback and student playtest data, ultimately resulting in the final version of the game, Lost at the Forever Mine, ${ }^{6}$ which was released on BrainPOP in October 2019 and received 6700 plays in the first six weeks.

Although the Wisconsin MRSEC created BREW to address both research exchange and engagement of MRSEC researchers in broader impacts, the BREW format can also be adapted for other groups. For example, departments or large laboratories could incorporate an education workshop into annual scientific retreats, or training grant directors could create a BREW-like event for their grantees. The education workshop can also focus on other science communication goals, such as creating blog posts or NSF highlights for public audiences, developing public engagement skills, or creating public elevator pitches on research topics. Our center has found that this fun, engaging workshop is an excellent way

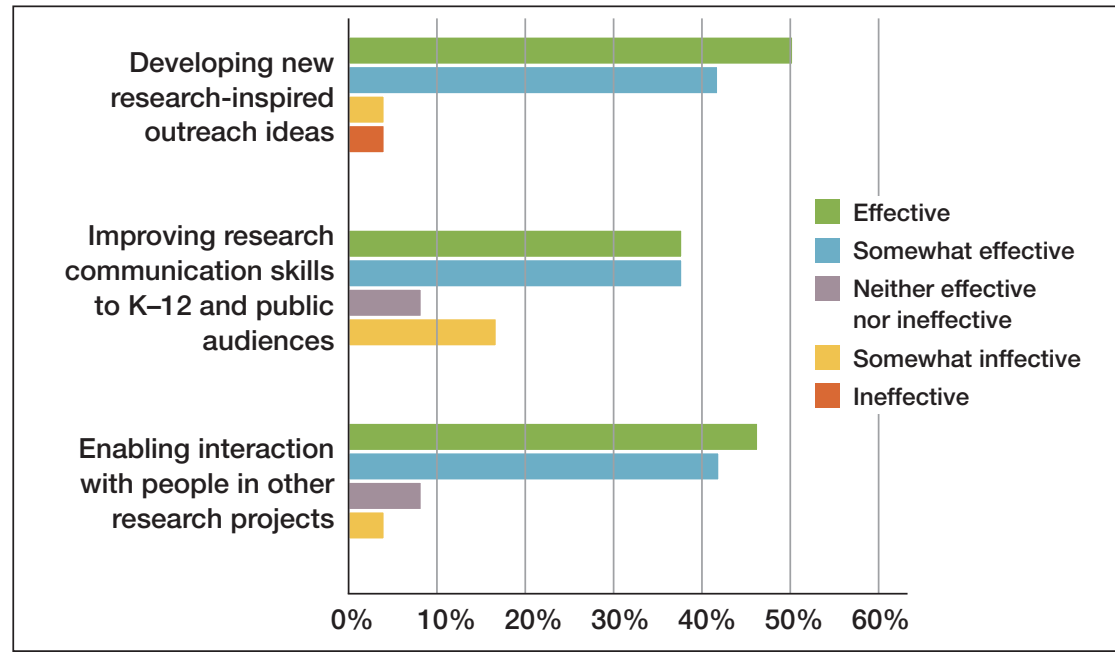

Figure 1. Survey responses from the 2013 and 2014 BREW E/O workshops $(n=24)$. to build community across our group and enhance all attendees' science communication skills, while addressing NSF's policy on Broader Impacts.

\section{Acknowledgments}

This work was primarily supported by NSF through the University of Wisconsin Materials Research Science

\section{University of Wisconsin MRSEC education \& outreach games}

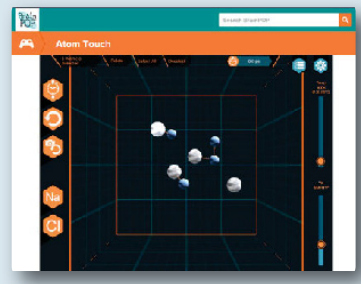

\section{Atom Touch}

\section{brainpop.com/games/atomtouch}

Explore atomic bonds. Change states of matter by adjusting temperature and pressure in this simulation that demonstrates how atoms move and bond.

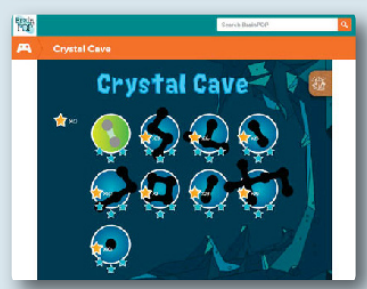

\section{Crystal Cave} brainpop.com/games/crystalcave

When the kids find a crystal cave under the Yard, they learn all about crystal molecules and dig up some sweet crystals for their collection in the museum.

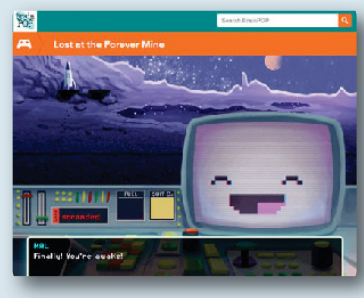

\section{Lost at the Forever Mine} brainpop.com/games/lostattheforevermine You just crash-landed on an abandoned mining planet! Use mathematical models to predict how to escape before time-and fuel-run out. and Engineering Center (DMR-1720415, DMR-1121288). Any opinions, findings, and conclusions or recommendations expressed in this report are those of the authors and do not necessarily reflect the views of the National Science Foundation.

\section{References}

1. National Science Foundation, NSF Proposal Processing and Review (February 25, 2019), https:// www.nsf.gov/pubs/policydocs/pappg19_1/pappg_3. jsp\#IIIA2b.

2. National Science Foundation, Broader Impacts Review Criterion (2007), https://www.nsf.gov/pubs/2007/ nsf07046/nsf07046.jsp.

3. Wisconsin MRSEC, Field Day Lab, "Atom Touch," BrainPOP(2018), https://www.brainpop.com/games/ atomtouch.

4. Wisconsin MRSEC, Field Day Lab, "Crystal Cave," BrainPOP(2018), https://www.brainpop.com/games/ crystalcave.

5. Achieve, Inc., Next Generation Science Standards: For States, By States, Topic Arrangements of the NGSS (2013), https://www.nextgenscience.org.

6. Wisconsin MRSEC, Field Day Lab, "Lost at the Forever Mine," BrainPOP(2019), https://www.brainpop.com/

\section{Further Reading}

1. P. Jensen, J. Rouquier, P. Kreimer, Y. Croissant, Sci. Public Policy 35 (7), 527 (2008).

2. E.L. Howell, J. Nepper, D. Brossard, M.A. Xenos, D.A. Scheufele, PLoS One 14, 5 (2019).

3. K. O'Meara, A. Jaeger, J. High. Educ. Outreach Engagem. 11 (4), 3 (2007).

We welcome comments and feedback on this article via email to Bulletin@mrs.org. games/lostattheforevermine. 